\title{
Direct amplitude detuning measurement with ac dipole
}

\author{
S. White \\ Brookhaven National Laboratory, Upton, New York 11973, USA \\ E. Maclean and R. Tomás \\ CERN, Geneva, Switzerland \\ (Received 21 May 2013; published 19 July 2013)
}

\begin{abstract}
In circular machines, nonlinear dynamics can impact parameters such as beam lifetime and could result in limitations on the performance reach of the accelerator. Assessing and understanding these effects in experiments is essential to confirm the accuracy of the magnetic model and improve the machine performance. A direct measurement of the machine nonlinearities can be obtained by characterizing the dependency of the tune as a function of the amplitude of oscillations (usually defined as amplitude detuning). The conventional technique is to excite the beam to large amplitudes with a single kick and derive the tune from turn-by-turn data acquired with beam position monitors. Although this provides a very precise tune measurement it has the significant disadvantage of being destructive. An alternative, nondestructive way of exciting large amplitude oscillations is to use an ac dipole. The perturbation Hamiltonian in the presence of an ac dipole excitation shows a distinct behavior compared to the free oscillations which should be correctly taken into account in the interpretation of experimental data. The use of an ac dipole for direct amplitude detuning measurement requires careful data processing allowing one to observe the natural tune of the machine; the feasibility of such a measurement is demonstrated using experimental data from the Large Hadron Collider. An experimental proof of the theoretical derivations based on measurements performed at injection energy is provided as well as an application of this technique at top energy using a large number of excitations on the same beam.
\end{abstract}

DOI: 10.1103/PhysRevSTAB.16.071002

PACS numbers: 41.85. $-\mathrm{p}, 29.27 .-\mathrm{a}, 29.20 .-\mathrm{c}$

\section{INTRODUCTION}

The recent start-up of the Large Hadron Collider (LHC) triggered renewed interest in nonlinear diagnostics for high-energy operation. Nonlinearities can be the source of resonance excitation and lead to a reduction of the dynamic aperture and poor beam lifetime. Although nonlinear field errors and related beam dynamics are generally optimized during the design phase of the accelerator, beam based diagnostics allow one to validate the models and their predictive capabilities for future upgrades. In addition, the efficiency of eventual corrections can be directly assessed by looking at the detuning coefficients. On the other hand, beam stability in the presence of collective effects such as impedance relies on Landau damping. Nonlinearities may be purposely introduced to mitigate these effects and possible interference with the machine nonlinearities could lead to inadequate magnet settings and misinterpretation of experimental results such as stability threshold.

A direct measurement of the machine nonlinearities can be obtained by characterizing the dependency of the tune

Published by the American Physical Society under the terms of the Creative Commons Attribution 3.0 License. Further distribution of this work must maintain attribution to the author(s) and the published article's title, journal citation, and DOI. as a function of the amplitude of oscillations. The conventional technique is to excite the beam to large amplitudes with a single kick and derive the tune from turn-by-turn data acquired with beam position monitors (BPMs). Although this provides a very precise tune measurement it has the significant disadvantage of being destructive and difficult to apply at high energies. An alternative, nondestructive way of exciting large amplitude oscillations is to use an ac dipole.

Linear optics measurements using an ac dipole have been successfully achieved in various machines [1-3]. The possibility of using an ac dipole for nonlinear diagnostics was described in [4,5] and a first experimental application measuring the resonance driving terms is described in [6]. Finally, an indirect amplitude detuning measurement was achieved at the Tevatron [2] using an ac dipole under some approximations described below.

However, past studies seem to assume that the effect of the ac dipole on the amplitude detuning is negligible and that one can approximate the motion undergoing forced oscillations to the case of free oscillations as long as linear optics distortions introduced by the ac dipole are small. In addition, the Tevatron studies [2] consist of an indirect measurement fitting the measured oscillation amplitude with theoretical expectations from the free oscillation model in the presence of a strong octupolar field and a drive frequency. 
The main objective of this paper is to present a new theory with experimental verification and demonstrate the feasibility of direct amplitude detuning measurement using an ac dipole as an efficient method to measure the machine nonlinearities at high energy. Although a comparison with the LHC nonlinear model is provided in Sec. VIIIC to assess the validity of the measurement, it is not our purpose to provide a detailed evaluation of the LHC multipole errors.

The first three sections are dedicated to the theory of particle motion undergoing driven oscillation. The perturbation Hamiltonian in the presence of an ac dipole excitation is derived and a distinct behavior compared to the free oscillations is demonstrated. It will be shown that the correction factors to be applied for the case of driven oscillation are well defined by the theory. A measurement of the tune as a function of the driven oscillation amplitude can therefore be considered as a direct amplitude detuning measurement. Section V presents the validation of the theory with tracking simulations and Sec. VI discusses the experimental protocol. An experimental verification of the theory at injection energy is presented in Sec. VII and a demonstration of the feasibility of the technique at high energy is presented in Sec. VIII.

\section{LINEAR MOTION WITH AC DIPOLE}

The Hamiltonian describing the linear motion of a single particle undergoing an ac dipole excitation can be expressed as [5]

$$
H_{0}\left(x, p_{x}, s, t\right)=\frac{1}{2} p_{x}^{2}+\frac{1}{2} K_{x}(s) x^{2}+\delta(s, t) x,
$$

where $x$ and $p_{x}$ are the transverse coordinates, $s$ the longitudinal coordinate, $K_{x}(s)$ is the focusing strength, and $\delta(t)$ is the time dependent kick from the ac dipole given by

$$
\delta(s, t)=\frac{q B L}{p} \delta_{\text {Dirac }}\left(s-s_{D}\right) \cos \left(2 \pi Q_{D} t+\varphi_{D}\right),
$$

where $B L$ is the integrated field amplitude, $q$ is the charge of the particle, $p$ its momentum, $s_{D}, Q_{D}$, and $\varphi_{D}$ are the location, the drive tune, and initial phase of the ac dipole, and $\delta_{\text {Dirac }}$ is the Dirac $\delta$ function. As shown in [4], an exact solution can be found for the particle motion at the location of the ac dipole for a given turn number $T$. Using the Courant-Snyder variables $\left(\hat{x}, \hat{p_{x}}\right)$ this solution can be written as

$$
\begin{aligned}
\hat{x}(T)-i \hat{p}_{x}(T)= & \sqrt{2 J_{x}} e^{i\left(2 \pi Q_{x} T+\psi_{x 0}\right)} \\
& +\delta_{-} e^{i 2 \pi Q_{D} T}-\delta_{+} e^{-i 2 \pi Q_{D} T},
\end{aligned}
$$

where $J_{x}$ and $\psi_{x 0}$ are the linear invariant and the phase given by the initial conditions of the test particle and depending on the adiabaticity of the ramping process of the ac dipole. $\delta_{ \pm}$is defined as

$$
\delta_{ \pm}=\sqrt{\beta_{D}} \frac{q B L}{p} \frac{e^{ \pm i\left(\pi Q_{ \pm}-\varphi_{D}\right)}}{4 \sin \left(\pi Q_{ \pm}\right)},
$$

where $\beta_{D}$ is the $\beta$ function at the ac dipole location and $Q_{ \pm}=Q_{D} \pm Q_{x}$ with $Q_{x}$ the natural tune. When the ac dipole is driven at a tune close to $Q_{x}$, which is generally the case, $\delta_{-}$is much larger than $\delta_{+}$and Eq. (3) can be reduced to

$$
\hat{x}(T)-i \hat{p}_{x}(T) \approx \sqrt{2 J_{x}} e^{i\left(2 \pi Q_{x} T+\psi_{x 0}\right)}+\delta_{-} e^{i 2 \pi Q_{D} T} .
$$

In the case of free oscillations, one can define the parametrized transverse coordinates $x$ and $y$, which will be used later in this paper

$$
\begin{aligned}
& x(s)=\sqrt{2 J_{x} \beta_{x}(s)} \cos \phi_{x}, \\
& y(s)=\sqrt{2 J_{y} \beta_{y}(s)} \cos \phi_{y},
\end{aligned}
$$

where $\phi_{x}$ and $\phi_{y}$ are the angle variables and $J_{x}$ and $J_{y}$ are the invariant of the motion. In case the ac dipole is driving the beam in the horizontal plane, an equivalent parametrization exists [2] and the $x$ coordinate becomes

$$
x_{D}(s)=\sqrt{2 J_{x} \beta_{x}(s)} \cos \phi_{x}+\sqrt{2 A \beta_{x}^{\prime}(s)} \cos \phi_{D},
$$

where $A$ and $\phi_{D}$ are the action and angle variables of the forced oscillations and $\beta^{\prime}(s)$ is the $\beta$ function modified by the ac dipole. Here we make the approximation that the forced oscillation term only depends on $\phi_{D}$ as was previously done in [5]. $J_{x}$ is determined by the adiabaticity of the ramping process of the ac dipole, i.e., for a perfectly adiabatic process $J_{x}=0$. In reality, the presence of nonlinearities or nonzero chromaticity will degrade the adiabaticity of the ramping process [7]. The term related to $J_{x}$ therefore appears and we could expect to observe a spectral line related to the natural tune when performing a frequency analysis of the beam motion.

The $\beta$ functions of a particle undergoing driven oscillation $\left(\beta^{\prime}\right)$ differs from those of the same particle undergoing free oscillation. A detailed analysis and computation of this effect can be found in [2]. The new $\beta$ functions of the driven particle are given by

$$
\beta^{\prime}(s)=\frac{1+\lambda_{D}^{2}-2 \lambda_{D} \cos \left(\psi_{D}\right)}{1-\lambda_{D}^{2}} \beta(s),
$$

where

$$
\lambda_{D}=\frac{\sin \left(\pi Q_{-}\right)}{\sin \left(2 \pi Q_{x}+\pi Q_{-}\right)},
$$

and $\psi_{D}$ relates to the phase advance with respect to the ac dipole location. As an example, typical parameters for the LHC collision optics are $Q_{x}=0.31$ and $Q_{-}=0.01$ which gives a peak $\left(\beta^{\prime}-\beta\right) / \beta$ due to the ac dipole of about $7 \%$. This difference can significantly vary depending on $Q_{x}$ and $Q_{-}$and should be reevaluated for other configurations. 


\section{AMPLITUDE DETUNING WITH OCTUPOLAR FIELD}

The magnetic field of a multipole of order $m$ is given by

$$
B_{y}(x, y, s)+i B_{x}(x, y, s)=\left[b_{m}(s)+i a_{m}(s)\right](x+i y)^{m-1},
$$

where the coefficients $b_{m}(s)$ and $a_{m}(s)$ are the so-called normal and skew coefficients expressed as

$$
\begin{aligned}
& b_{m}(s)=\left.\frac{1}{(m-1) !} \frac{\partial^{m-1} B_{y}}{\partial x^{m-1}}\right|_{(0,0, s)}, \\
& a_{m}(s)=\left.\frac{1}{(m-1) !} \frac{\partial^{m-1} B_{x}}{\partial x^{m-1}}\right|_{(0,0, s)} .
\end{aligned}
$$

This leads to the expression of the total Hamiltonian which becomes $H_{0}+H_{p}$ where $H_{p}$ is the perturbation Hamiltonian expressed as

$$
H_{p}=\frac{q}{p} \operatorname{Re}\left[\sum_{m=3}^{\infty} \frac{1}{m}\left[b_{m}(s)+i a_{m}(s)\right](x+i y)^{m}\right] .
$$

In the following, we will neglect all the contributions from $x / y$ coupling. For clarity we introduce the variable $H_{2 n}$ corresponding to the terms of the perturbation Hamiltonian related to the multipole of order $2 n$. When the beam is oscillating at reasonable amplitudes the detuning is dominated by the octupolar term. Expanding $H_{p}$ and keeping only the $b_{4}$ terms the perturbation Hamiltonian $H_{4}$ for a normal octupole $(n=2)$ is given by

$$
H_{4}=\frac{q}{p} \frac{b_{4}(s)}{4}\left(x^{4}-6 x^{2} y^{2}+y^{4}\right),
$$

where $x$ and $y$ come from Eqs. (7) and (8). The detuning can be derived analytically by averaging the perturbation Hamiltonian over all the phase variables and computing the integrals,

$$
\Delta Q_{x, y}=\frac{1}{2 \pi} \oint \frac{\partial}{\partial J_{x, y}}\left\langle H_{2 n}\right\rangle d s .
$$

Inserting Eqs. (7) and (8) into Eq. (15) and making use of the properties of the cosine function we get

$$
\begin{aligned}
\left\langle H_{4}\right\rangle= & \frac{q}{p} \frac{b_{4}(s)}{4}\left[\frac{3}{2} \beta_{x}(s)^{2} J_{x}^{2}+\frac{3}{2} \beta_{y}(s)^{2} J_{y}^{2}+\frac{3}{2} \beta_{x}^{\prime}(s)^{2} A^{2}\right. \\
& +6 \beta_{x}(s) \beta_{x}^{\prime}(s) J_{x} A-6 \beta_{x}(s) \beta_{y}(s) J_{x} J_{y} \\
& \left.-6 \beta_{x}^{\prime}(s) \beta_{y}(s) A J_{y}\right] .
\end{aligned}
$$

Under the approximation that the $\beta$ functions and $b_{4}$ are constant over the length of the magnet, this leads to the expressions of the amplitude dependent tunes,

$$
\Delta Q_{x}=\frac{q}{p} \frac{3 B_{4}}{8 \pi}\left(\beta_{x}^{2} J_{x}+2 \beta_{x} \beta_{x}^{\prime} A-2 \beta_{x} \beta_{y} J_{y}\right)
$$

$$
\Delta Q_{y}=\frac{q}{p} \frac{3 B_{4}}{8 \pi}\left(\beta_{y}^{2} J_{y}-2 \beta_{y} \beta_{x}^{\prime} A-2 \beta_{x} \beta_{y} J_{x}\right),
$$

where $B_{4}$ is the coefficient of the integrated field. The amplitude detuning for a purely horizontal ac dipole excitation $\left(J_{y}=0\right)$ is then expressed as

$$
\begin{gathered}
\Delta Q_{x}=\frac{q}{p} \frac{3 B_{4}}{8 \pi}\left(\beta_{x}^{2} J_{x}+2 \beta_{x} \beta_{x}^{\prime} A\right), \\
\Delta Q_{y}=-\frac{q}{p} \frac{3 B_{4}}{8 \pi}\left(2 \beta_{x}^{\prime} \beta_{y} A+2 \beta_{x} \beta_{y} J_{x}\right) .
\end{gathered}
$$

From these expressions it is seen that there is a distinct behavior of the amplitude detuning for free oscillations $\left(A=0, \Delta Q_{x} \propto \beta_{x}^{2} J_{x}\right)$ and for driven oscillations $\left(A \gg J_{x}\right.$, $\left.\Delta Q_{x} \propto 2 \beta_{x} \beta_{x}^{\prime} A\right)$. Assuming $\beta^{\prime} \approx \beta$ the direct term $\Delta Q_{x}$ is larger by a factor 2 while the cross term $\Delta Q_{y}$ is not affected. This will have to be taken into account when analyzing the data.

\section{GENERALIZATION TO MULTIPOLES OF ORDER $\boldsymbol{m}=\mathbf{2 n}$}

Although it is more difficult to measure the contribution of multipoles of order higher than the octupole, it is interesting to generalize the above calculations. Multipoles of order $2 n+1$ will not contribute to the first order amplitude detuning. We will therefore restrict ourselves to the calculation for multipoles of order $2 n$. The average value of a cosine to the powers $2 n$ and $2 n+1$ can be expressed as

$$
\left\langle\cos (x)^{2 n}\right\rangle=2^{-2 n}\left(\begin{array}{c}
2 n \\
n
\end{array}\right), \quad\left\langle\cos (x)^{2 n+1}\right\rangle=0,
$$

where

$$
\left(\begin{array}{c}
2 n \\
n
\end{array}\right)
$$

is the binomial coefficient $C_{2 n}^{n}$. From Eq. (14), the perturbation Hamiltonian of a normal multipole of order $2 n$ is expressed as

$$
H_{2 n}=\frac{q b_{2 n}}{2 n p} \operatorname{Re}\left[(x+i y)^{2 n}\right]=\frac{q b_{2 n}}{2 n p} \sum_{k=0}^{n}\left(\begin{array}{l}
2 n \\
2 k
\end{array}\right) x^{2 n-2 k} i^{2 k} y^{2 k} .
$$

Using Eq. (22) the average Hamiltonian is given by

$$
\begin{aligned}
\left\langle H_{2 n}\right\rangle= & \frac{q b_{2 n}}{2 n p} \sum_{k=0}^{n} 2^{-n}\left(\begin{array}{c}
2 n \\
2 k
\end{array}\right)\left(\begin{array}{c}
2 n-2 k \\
n-k
\end{array}\right) \\
& \times\left(\begin{array}{c}
2 k \\
k
\end{array}\right) i^{2 k} \beta_{x}(s)^{n-k} \beta_{y}(s)^{k} J_{x}^{n-k} J_{y}^{k} .
\end{aligned}
$$

The amplitude dependent tunes can be calculated using Eq. (16) and are expressed as 


$$
\begin{aligned}
\Delta Q_{x}= & \frac{q B_{2 n}}{2 n p} \frac{1}{2 \pi} \sum_{k=0}^{n-1} 2^{-n}\left(\begin{array}{c}
2 n \\
2 k
\end{array}\right)\left(\begin{array}{c}
2 n-2 k \\
n-k
\end{array}\right) \\
& \times\left(\begin{array}{c}
2 k \\
k
\end{array}\right) i^{2 k} \beta_{x}^{n-k} \beta_{y}^{k}(n-k) J_{x}^{n-k-1} J_{y}^{k}, \\
\Delta Q_{y}= & \frac{q B_{2 n}}{2 n p} \frac{1}{2 \pi} \sum_{k=1}^{n} 2^{-n}\left(\begin{array}{c}
2 n \\
2 k
\end{array}\right)\left(\begin{array}{c}
2 n-2 k \\
n-k
\end{array}\right) \\
& \times\left(\begin{array}{c}
2 k \\
k
\end{array}\right) i^{2 k} \beta_{x}^{n-k} \beta_{y}^{k} J_{x}^{n-k} k J_{y}^{k-1} .
\end{aligned}
$$

One can see from these expressions that for multipoles of order $2 n>4$ the direct and cross term of the amplitude detuning will not strictly depend on $J_{x}$ and $J_{y}$ as was the case for $2 n=4$. However, when measuring the amplitude detuning, only one plane is generally excited. Under the assumption that coupling is negligible we have $J_{x} \gg J_{y}$ in case of horizontal excitation and can make the approximation that all the terms depending on $J_{y}$ are negligible. The amplitude dependent tunes are then reduced to

$$
\begin{gathered}
\Delta Q_{x}=\frac{q B_{2 n}}{2 n p} \frac{2^{-n}}{2 \pi}\left(\begin{array}{c}
2 n \\
0
\end{array}\right)\left(\begin{array}{c}
2 n \\
n
\end{array}\right) \beta_{x}^{n} n J_{x}^{n-1} \\
=\frac{q B_{2 n}}{2 n p} \frac{2^{-n}}{2 \pi} \frac{(2 n) !}{(n-1) ! n !} \beta_{x}^{n} J_{x}^{n-1}, \\
\Delta Q_{y}=-\frac{q B_{2 n}}{2 n p} \frac{2^{-n+1}}{2 \pi}\left(\begin{array}{c}
2 n \\
2
\end{array}\right)\left(\begin{array}{c}
2 n-2 \\
n-1
\end{array}\right) \beta_{x}^{n-1} \beta_{y} J_{x}^{n-1} \\
=-\frac{q B_{2 n}}{2 n p} \frac{2^{-n}}{2 \pi} \frac{(2 n) !}{(n-1) !(n-1) !} \beta_{x}^{n-1} \beta_{y} J_{x}^{n-1} .
\end{gathered}
$$

When the beam is excited by an ac dipole instead of a single kick, the variable $x$ has to be replaced by $x_{D}$ as defined in Eq. (8). The averaged Hamiltonian in Eq. (24) now becomes

$$
\left\langle H_{2 n}\right\rangle=\frac{q b_{2 n}}{2 n p} \sum_{k=0}^{n}\left(\begin{array}{c}
2 n \\
2 k
\end{array}\right)\left(\begin{array}{c}
2 k \\
k
\end{array}\right) 2^{-k} i^{2 k} \beta_{y}(s)^{k} J_{y}^{k}\left\langle x_{D}^{2 n-2 k}\right\rangle,
$$

where

$$
\begin{aligned}
\left\langle x_{D}^{2 n-2 k}\right\rangle= & \sum_{l=0}^{n-k}\left(\begin{array}{c}
2 n-2 k \\
2 l
\end{array}\right)\left(\begin{array}{c}
2 n-2 k-2 l \\
n-k-l
\end{array}\right) \\
& \times\left(\begin{array}{c}
2 l \\
l
\end{array}\right) 2^{-n+k} \beta_{x}(s)^{n-k-l} \beta_{x}^{\prime}(s)^{l} J_{x}^{n-k-l} A^{l},
\end{aligned}
$$

where only even powers of $l$ were retained, odd powers vanish due to the averaged cosine. The amplitude dependent tunes in the presence of an ac dipole excitation are again computed using Eq. (16) and are expressed as

$$
\begin{aligned}
\Delta Q_{x}= & \frac{q B_{2 n}}{2 n p} \frac{1}{2 \pi} \sum_{k=0}^{n-1}\left(\begin{array}{c}
2 n \\
2 k
\end{array}\right)\left(\begin{array}{c}
2 k \\
k
\end{array}\right) 2^{-n} i^{2 k} \beta_{y}^{k} J_{y}^{k} \\
& \times \sum_{l=0}^{n-k-1}\left(\begin{array}{c}
2 n-2 k \\
2 l
\end{array}\right)\left(\begin{array}{c}
2 n-2 k-2 l \\
n-k-l
\end{array}\right) \\
& \times\left(\begin{array}{c}
2 l \\
l
\end{array}\right) \beta_{x}^{n-k-l} \beta_{x}^{l l}(n-k-l) J_{x}^{n-k-l-1} A^{l}, \\
\Delta Q_{y}= & \frac{q B_{2 n}}{2 n p} \frac{1}{2 \pi} \sum_{k=1}^{n}\left(\begin{array}{c}
2 n \\
2 k
\end{array}\right)\left(\begin{array}{c}
2 k \\
k
\end{array}\right) 2^{-n} i^{2 k} \beta_{y}^{k} k J_{y}^{k-1} \\
& \times \sum_{l=0}^{n-k}\left(\begin{array}{c}
2 n-2 k \\
2 l
\end{array}\right)\left(\begin{array}{c}
2 n-2 k-2 l \\
n-k-l
\end{array}\right) \\
& \times\left(\begin{array}{c}
2 l \\
l
\end{array}\right) \beta_{x}^{n-k-l} \beta_{x}^{\prime l} J_{x}^{n-k-l} A^{l} .
\end{aligned}
$$

In the case of free oscillations, we have $A=0$ and only the terms for $l=0$ survive, in which case we recover Eqs. (25) and (26). When amplitude detuning is measured with the ac dipole we have $A \gg J_{x}$ and $A \gg J_{y}$ and make the approximation that all the terms depending on $J_{x}$ and $J_{y}$ are negligible. In this case the amplitude dependent tunes are reduced to

$$
\begin{aligned}
\Delta Q_{x} & =\frac{q B_{2 n}}{2 n p} \frac{2^{-n+1}}{2 \pi}\left(\begin{array}{c}
2 n \\
2 n-2
\end{array}\right)\left(\begin{array}{c}
2 n-2 \\
n-1
\end{array}\right) \beta_{x} \beta_{x}^{\prime n-1} A^{n-1} \\
& =\frac{q B_{2 n}}{2 n p} \frac{2^{-n}}{2 \pi} \frac{(2 n) !}{(n-1) !(n-1) !} \beta_{x} \beta_{x}^{\prime n-1} A^{n-1} \\
\Delta Q_{y} & =-\frac{q B_{2 n}}{2 n p} \frac{2^{-n+1}}{2 \pi}\left(\begin{array}{c}
2 n \\
2
\end{array}\right)\left(\begin{array}{c}
2 n-2 \\
n-1
\end{array}\right) \beta_{y} \beta_{x}^{\prime n-1} A^{n-1} \\
& =-\frac{q B_{2 n}}{2 n p} \frac{2^{-n}}{2 \pi} \frac{(2 n) !}{(n-1) !(n-1) !} \beta_{y} \beta_{x}^{\prime n-1} A^{n-1}
\end{aligned}
$$

Using $n=2$ in Eqs. (27), (28), (33), and (34) we recover the free oscillation and ac dipole terms for an octupolar field derived in the previous section. Assuming $\beta_{x}^{\prime}=\beta_{x}$ we can conclude, from the comparison of the derivation for free oscillation, Eqs. (27) and (28), and ac dipole, Eqs. (33) and (34), that for multipoles of order $2 n$, the direct term of the amplitude detuning measured with an ac dipole will be a factor $n$ larger than what is expected for free oscillations while the cross terms in both cases are equal. Under the approximation $\beta_{x}^{\prime}=\beta_{x}$ the correction factors to be applied when measuring the detuning as a function of $A$ are therefore well defined and the measurement of amplitude detuning with ac dipole can therefore still be considered as direct.

It should be noted that these theoretical derivations do not include any second order effects. In reality, second order effects can have a sizable effect and should be taken into account when comparing the model and experimental 
data. The comparison between the model and experimental data presented in Sec. VIII was performed by tracking the full LHC lattice in MAD-X [8] which takes into account higher order terms. Table II provides a direct comparison between the free oscillation and ac dipole models and confirms the theoretical expectations. This indicates that no significant additional discrepancies between free and forced oscillations amplitude detuning measurements should be expected from second order effects.

\section{SINGLE PARTICLE TRACKING}

In order to validate the above calculations a simple tracking code was built. A single particle is tracked through a linear betatron map to which an ac dipole and an arbitrary number of multipoles of order $2 n$ are added. This simple tracking code was benchmarked with MAD-X in the case of a single octupole as the only source of nonlinearity. The tunes at zero amplitude used in the following examples are $Q_{x}=0.31$ and $Q_{y}=0.32$ and the excitation is applied in the horizontal plane only with $Q_{-}=0.01$. The ac dipole ramp and flattop both last 2000 turns. A small initial amplitude was applied in the vertical plane in order to measure the tune from the turn-by-turn data. The model uses Eqs. (33) and (34) where the effect of the ac dipole on the $\beta$ function was also included. The amplitude of oscillations corresponds to half the peak-to-peak amplitude of the turn-by-turn data over the ac dipole flattop and the natural tunes are computed using an interpolated fast Fourier transform (FFT) over the whole ac dipole flattop. The tracking was performed up to unrealistic amplitudes and the value of the field components were set in order to clearly observe the effect of higher order multipoles.

The results of the single particle tracking for a single multipole of order $2 n$ are shown in Figs. 1 and 2. Even though we assumed that all the $J_{x}$ and $J_{y}$ terms are

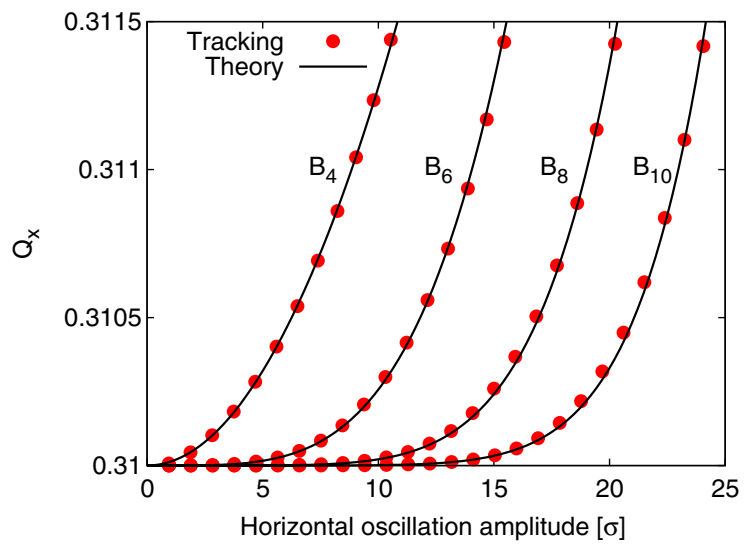

FIG. 1. Direct term of amplitude detuning for a single multipole of order $2 n$. The red dots are the result of the tracking and the black lines are the theoretical predictions for an ac dipole excitation (free oscillations term corrected by $n$ ).

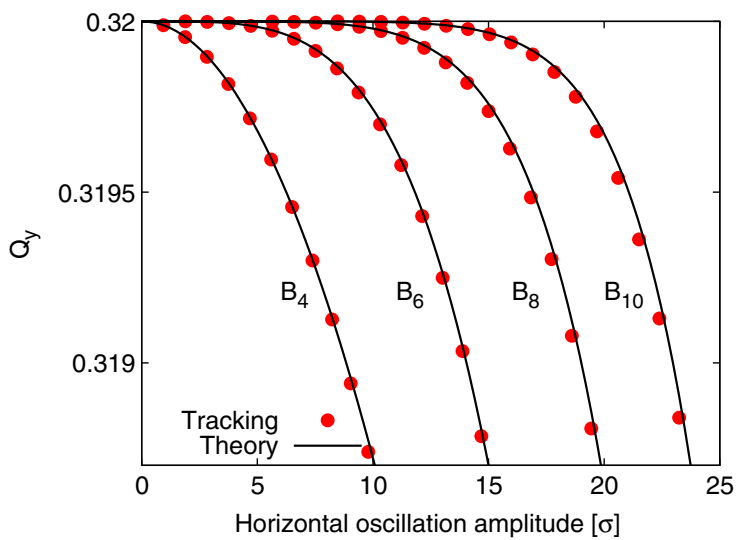

FIG. 2. Cross term of amplitude detuning for a single multipole of order $2 n$. The red dots are the result of the tracking and the black lines are the theoretical predictions for an ac dipole excitation (equal to the free oscillations term).

negligible, tracking data are in excellent agreement with theoretical predictions up to a $B_{10}$ field component. This confirms that the direct amplitude detuning from a multipole of order $2 n$ field has to be corrected by a factor $n$ when measured with an ac dipole while the cross term is directly measured.

As shown in Figs. 3 and 4, the behavior for the combined effect of nonlinear field components up to $B_{10}$ is similar to the one for a single multipole. The cross term is consistent with the free oscillation model whereas the coefficients of the direct term have to be corrected by a factor $n$ in order to reach agreement. Small discrepancies are observed at large amplitudes which could be due to higher order effects or the approximations made in the model. These are not so relevant in real experiments.

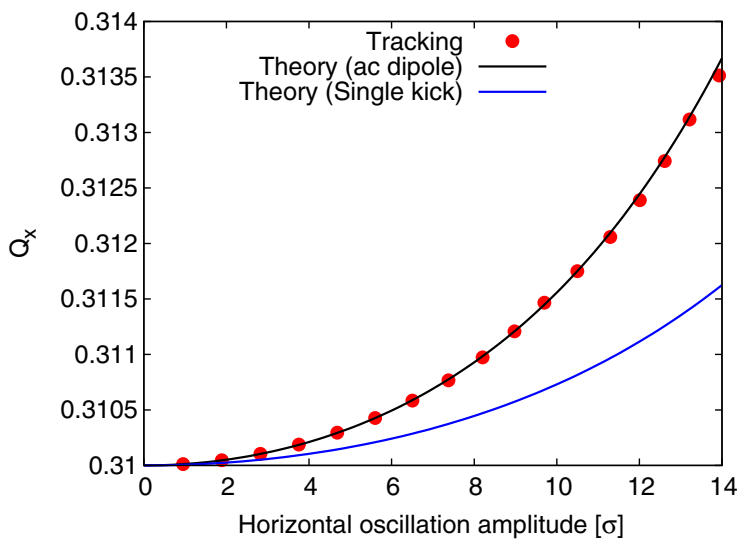

FIG. 3. Direct term of amplitude detuning for the combined effect of nonlinear field components up to $B_{10}$. The red dots are the result of the tracking, the blue line is the model prediction for free oscillation, and the black line represents the model with all coefficients rescaled by $n$. Tracking data are consistent with the model for free oscillation corrected by a factor $n$. 


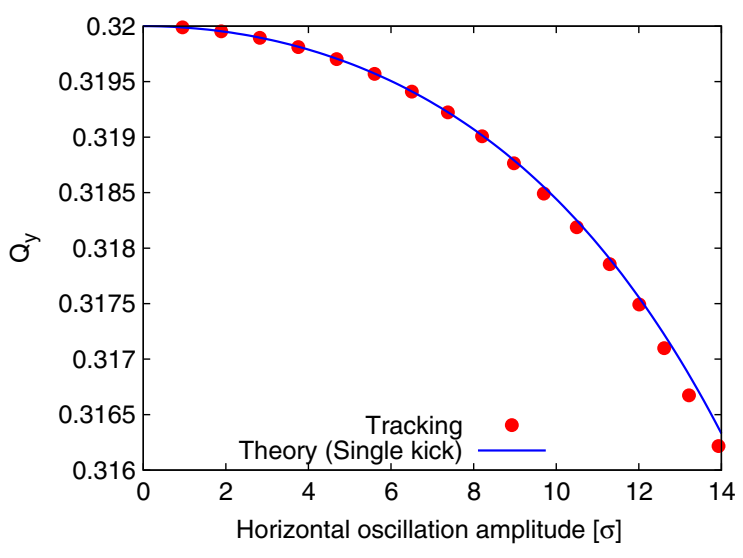

FIG. 4. Cross term of amplitude detuning for the combined effect of nonlinear field components up to $B_{10}$. The red dots are the result of the tracking; the blue line is the model prediction for free oscillation. Tracking data are consistent with the free oscillation term.

\section{DATA PROCESSING AND ANALYSIS}

Approximately 500 BPMs are spread around each of the LHC rings providing as many independent signals to be combined for frequency analysis. In order to compute the amplitude detuning both the action and the natural tune must be derived from the turn-by-turn data. This is done using an analysis package developed for the LHC. A description can be found in [3,9]. The action is derived from the measured $\beta$ functions and represents the average of all BPMs in the ring. The natural tune measurement is extracted from a frequency analysis of the LHC BPMs and will be described in more detail in the following section.

BPMs could be malfunctioning or subject to noise that can degrade the measured signals. This is particularly relevant in the case of an ac dipole excitation where the frequency spectrum is dominated by the drive frequency. The natural tune peak amplitude is typically a few orders of magnitude smaller than the ac dipole peak. The first step in the analysis is therefore to perform a singular value decomposition (SVD) analysis allowing to remove the faulty BPMs and retain only the real physical modes of the beam, removing those related to noise as described in $[10]$.

The SVD of an arbitrary matrix $M$ is given by

$$
M=U \Sigma V^{T}=\sum_{i} u_{i} \sigma_{i} v_{i}^{T},
$$

where $u_{i}$ and $v_{i}$ are the normalized eigenvectors of $M M^{T}$ and $M^{T} M$, respectively. $\Sigma$ is a diagonal matrix of singular values $\sigma_{i}$. Performing an SVD analysis of a matrix combining the turn-by-turn data from all ring BPMs, one can therefore extract a collection of singular vectors characterizing the temporal and spatial variation of modes, which describe the beam motion. Only the dominant modes are representative of the real physical motion of the beam.
Performing a truncation over the singular values and inverting the SVD transformation therefore allows one to restore the turn-by-turn data from which the irrelevant modes are removed, and hence reduce the noise floor of the frequency spectrum. We can define the SVD cut as the number of singular values considered for the analysis.

The effect of the SVD preprocessing of the data is illustrated in Fig. 5 where it is seen that an SVD cut of 10 is necessary to measure a clean natural tune signal above the noise floor. The data set shown on this plot is for an excitation amplitude of $1 \sigma$. Once the data is cleaned, the natural tune seen by each of the available BPMs is computed using an interpolated FFT algorithm. The global tune of the machine and its statistical error bar are derived from the mean and standard deviation of these values. Typical tune distributions obtained for the LHC are shown in Fig. 6 where it is seen that a proper cleaning of the raw turn-by-turn data not only allows one to observe the natural tune but also significantly improves the resolution of the measurement.

As mentioned in Sec. II, the observation of the natural tune spectral line relies on the fact that the ac dipole is not perfectly adiabatic. The nonadiabaticity of the ramping process is mostly driven by the machine nonlinearities and should increase with the oscillation amplitude. In case of strong nonadiabaticity, the approximation $J_{x} \ll A$ does not hold anymore and the theoretical derivations presented in Sec. IV cannot be applied. The relative amplitudes of the natural tune and drive frequency spectral lines give a good measurement of the importance of this effect. This is illustrated on the top plot of Fig. 7 where the ratio of these spectral lines in both planes is shown as a function of the horizontal oscillation amplitude. The ratio in the horizontal plane, in which the ac dipole kick strength

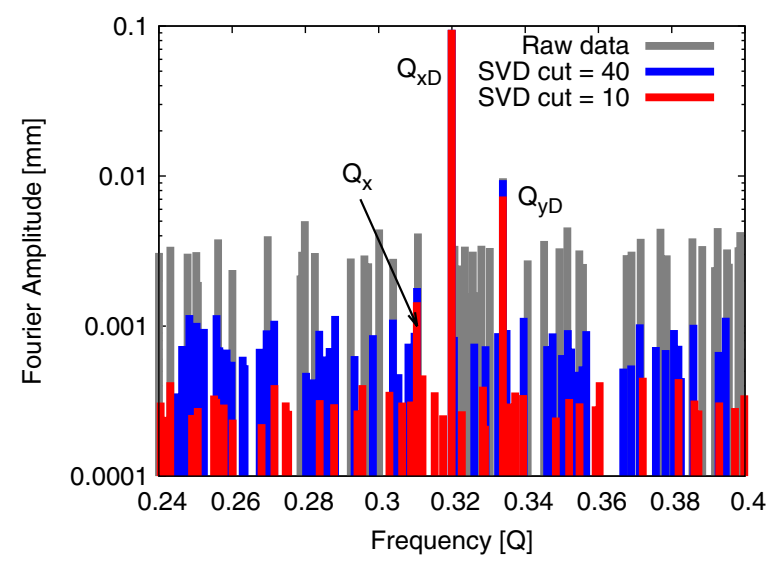

FIG. 5. Frequency spectrum of an LHC arc BPM with an oscillation amplitude of $1 \sigma$. Both horizontal and vertical ac dipole drive frequencies $\left(Q_{x D}\right.$ and $\left.Q_{y D}\right)$ are observed in the spectrum. The natural tune is within the noise floor for the raw data, barely above for an SVD cut of 40 and about a factor 5 above for an SVD cut of 10 . 


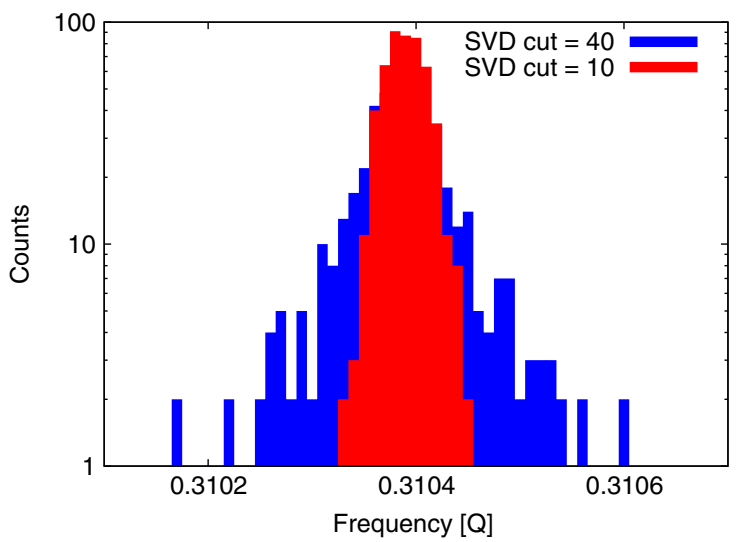

FIG. 6. Tune distribution of all LHC BPMs for SVD cuts of 10 and 40 .

was scanned, remains constant indicating that the nonadiabaticity of the ramping process approximately scales with the oscillation amplitude. In the vertical plane, although the driven $Q_{y}$ action is constant as shown on the bottom plot of Fig. 7, this ratio increases during the scan. This increase can therefore be attributed to coupling, i.e., the horizontal ac dipole also excites the vertical plane.

The example shown here is for data taken at top energy presented in Sec. VIII. This verification was performed for all the data sets presented in this paper and it was found that in all cases the natural tune amplitude is within a few percents of the drive frequency amplitude. The net effect on the total action can therefore be considered as negligible and should not affect the amplitude detuning measurement.
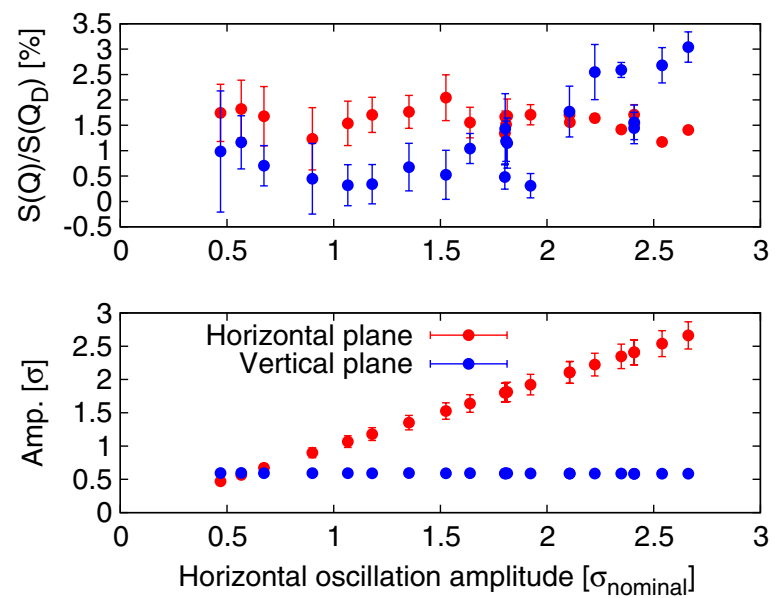

FIG. 7. On the top the ratio of the amplitudes of the drive tune and natural tune spectral lines in the horizontal and vertical plane is shown. Each plane is treated separately. On the bottom plot the oscillation amplitudes in both planes are shown as a function of the horizontal oscillation amplitude. This data set was acquired at top energy.

\section{EXPERIMENTAL VERIFICATION OF THE THEORY}

Nonlinear beam optics measurements were performed for Beam 2 at the LHC injection energy $(450 \mathrm{GeV})$ during which both the ac dipole and a single kick were used to excite the beam [11]. Comparing the detuning coefficients measurements with both methods provides a direct validation of the theoretical derivations from Secs. III and IV. The original objective of these experiments was not to measure amplitude detuning with ac dipole or demonstrate the related theory. Only one useful data set could be extracted from these experiments for this purpose. The results are shown in Fig. 8 where a distinct behavior of the amplitude detuning measured with the ac dipole and a single kick is clearly observed.

Table I summarizes the results obtained with ac dipole excitation and single kick excitation. In this example the detuning is dominated by the octupolar term and a simple parabolic fit was applied to find the coefficients. The uncertainties shown in the first two rows of Table I account only for the statistical error bars from the parabolic fits. The theoretical derivations in Secs. III and IV were done under the approximation that $\beta^{\prime}=\beta$. Applying Eq. (9) to the experimental conditions $\left(Q_{x}=0.28\right.$ and $\left.Q_{-}=0.01\right)$, one can derive an upper limit of $6 \%$ for the systematic error to be applied to theoretical predictions from Sec. III. The measured direct term of the detuning differs by a factor $2.07 \pm 5.0 \%$ and the cross terms by a factor $0.95 \pm 13.2 \%$ while the theory predicts ratios of 2.0 for the direct term and 1.0 for the cross term in the case of octupolar detuning. The measured values are therefore consistent with theoretical expectation. This constitutes the experimental verification of the theory presented in this paper.
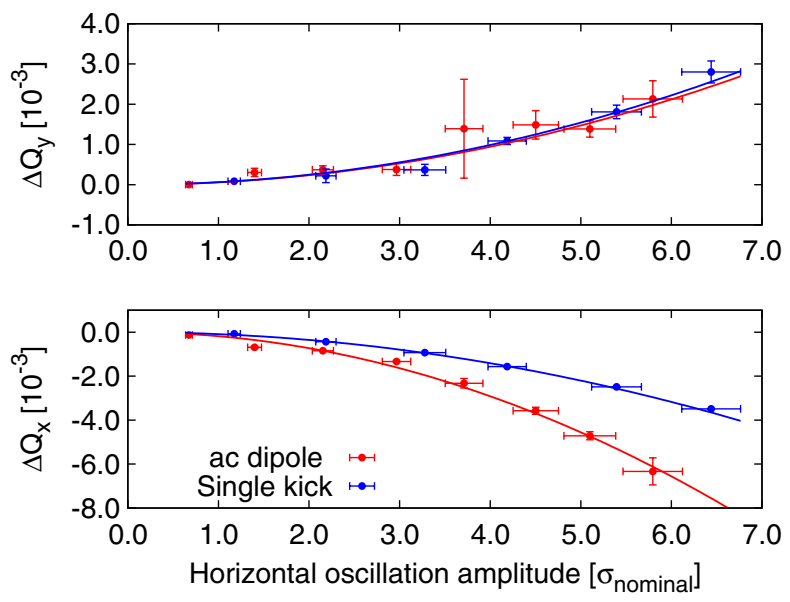

FIG. 8. Direct and cross term of the amplitude detuning at injection measured using a single kick or an ac dipole excitation for Beam 2. $\sigma_{\text {nominal }}$ represents the LHC nominal rms beam size for an emittance of $3.75 \mu \mathrm{m}$. The plain lines represent a parabolic fit of the data points. 
TABLE I. Octupolar term given by the fit results ac dipole and single kick data at injection energy. The errors given in this table represent the fit errors. The predicted ratio represents theoretical expectations for octupolar detuning.

\begin{tabular}{lcc}
\hline \hline & $\partial Q_{x} / \partial 2 J_{x}\left[\mathrm{~m}^{-1}\right]$ & $\partial Q_{y} / \partial 2 J_{x}\left[\mathrm{~m}^{-1}\right]$ \\
\hline ac dipole & $-20507 \pm 4.0 \%$ & $6627 \pm 11.5 \%$ \\
Free oscillation & $-9918 \pm 3.0 \%$ & $6944 \pm 6.5 \%$ \\
Measured ratio & $2.07 \pm 5.0 \%$ & $0.95 \pm 13.2 \%$ \\
Predicted ratio & 2.00 & 1.00 \\
\hline \hline
\end{tabular}

Detailed nonlinear optics measurements at injection energy and comparison with the LHC nonlinear model can be found in [12]. Amplitude detuning measurements based on single kick data were performed before and after nonlinear optics corrections. The measurements and the model agree within 10\%-20\% for nominal injection settings (strong octupoles).

\section{APPLICATION AT TOP ENERGY}

Amplitude detuning measurements with ac dipole were performed at the LHC top energy of $4 \mathrm{TeV}$ [13]. Squeezed optics with $\beta^{*}=0.6 \mathrm{~m}$ in IR1 and IR5 (IR stands for insertion region) were used for these measurements. The experimental data presented in this section were acquired during the same fill taking full advantage of the nondestructive properties of ac dipole excitation. Since it was not possible to acquire single kick data during this experiment, the experimental results are compared to the model. A detailed description of how the model was built is therefore provided in this section. Difficulties related to nonlinear resonances encountered during this measurement will be discussed.

\section{A. Modeling the LHC in MAD-X}

The model of the LHC has been developed using MAD-X and includes our best knowledge of the nonlinear sources. The model is constructed using the thin lens approximation, to which the measured magnetic errors (generated by the Windows Interface to Simulation Errors, WISE [14]) of order $\left(B_{3}, A_{3}\right)$ up to $\left(B_{15}, A_{15}\right)$ in the main bends (MB), main quadrupoles, insertion quadrupoles, and insertion dipoles are applied. The $B_{2}$ and $A_{2}$ errors are assumed to be well corrected. The spool piece magnets (which correct for the errors in the MB), and the IR correctors are set in the model to reproduce the conditions during the measurement. The status of the nonlinear correctors in the IRs during the measurement was with corrections applied for the sextupolar and normal-octupolar errors in IR1, and the sextupolar errors in IR5. The status of the spool pieces was nominal operational settings with the exception of malfunctioning $B_{4}$ circuits in three arcs, which were at zero field. The crossing angles and separation bumps present
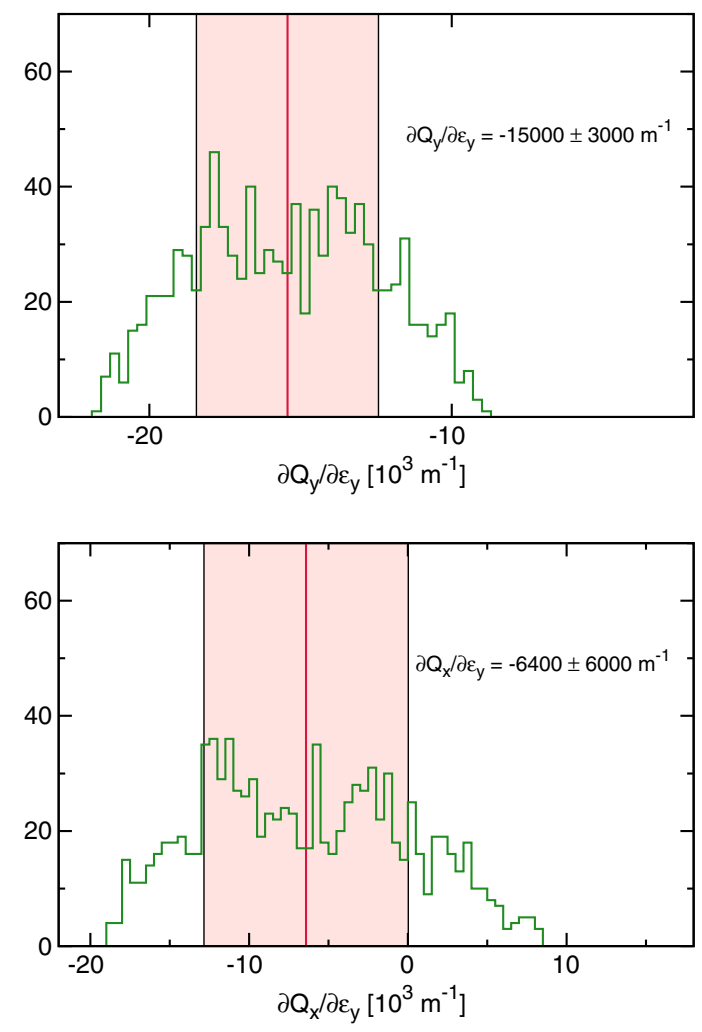

FIG. 9. The distribution of anharmonicities obtained from PTC for 1000 instances of a MAD-X model of the LHC based on our best knowledge of the nonlinear sources and including random distribution of coupling amplitude and phase. 
during the measurements are incorporated. The orbit is corrected to zero and the coupling, tune, and chromaticity are matched to the measured values. The detuning may then be determined using the PTC_NORMAL module in MAD-X [15], or from tracking, to enable a comparison with the measured data.

The dominant source of the uncertainty in the model seems to be the amplitude and phase of the linear coupling. While we may characterize the coupling by the value measured with the LHC base band tune (BBQ) system [16], local sources mean the amplitude and phase of the linear coupling resonance driving terms vary around the ring. As yet we do not have a coupling model of the LHC which accurately reproduces this variation. To assess the uncertainty in the modeled anharmonicities due to the linear coupling, the matching targets of the coupling amplitude and phase were varied. The coupling amplitude was varied randomly with a Gaussian distribution of $\sigma=0.002$ centered on 0.003 (the value measured by the BBQ), and truncated at $1 \sigma$. The coupling phase was varied randomly on an even distribution between 0 and $2 \pi$.

From these random parameters we produced 1000 instances of the LHC, and analyzed the detuning in each case. The results are presented in Fig. 9.

We observe a substantial distribution in the measurements, with a standard deviation of approximately $20 \%$ for the term $\partial Q_{x} / \partial \epsilon_{x}$ and of approximately $\sim 100 \%$ for the term $\partial Q_{y} / \partial \epsilon_{x}$. These results were used as uncertainties in Fig. 13 where the measurements are compared to the model.

It is worth recalling that during these measurements the dominant sources of amplitude dependent detuning in the LHC (notably the Landau octupoles and the octupolar errors in the arc dipoles and the triplets) were either switched off or partially corrected. This leaves the smaller and less well understood errors to drive the detuning: this clearly limits our ability to accurately reproduce the nonlinear optics during these measurements. Other sources of uncertainty related to misalignments and orbit effects may also apply. These sources have not been included in these simulations due to technical limitations with MAD-X and PTC.

\section{B. Impact of nonlinear resonances}

In some cases, nonlinear resonances can be excited and bias the analysis. These resonances are described by [5,7]

$$
n Q_{x}+m Q_{y}+l Q_{x D}+k Q_{y D}=u,
$$

where $n, m, k, l$, and $u$ are integers and $n$ and $m$ cannot be equal to zero at the same time. It is seen that in the presence of dual plane ac dipole excitation four different frequencies can be used to build resonance conditions whereas only $Q_{x}$ and $Q_{y}$ are relevant for free oscillations. Nonlinear resonances were mainly observed when trying to measure the cross term of the amplitude detuning for which the excitation is provided by the other plane. In some cases, the natural tune signal was weaker than some other spectral lines associated with nonlinear resonances resulting in the peak finder locking onto the wrong spectral line.

Figure 10 shows a comparison between the direct term, where the tune distribution of all BPMs is described by a single peak, and the cross term which is much more noisy and for which several peaks are observed even with a very aggressive cleaning. The example shown here is a particularly bad data point and is not representative of what is generally observed. One could simply exclude these bad data points from the final analysis but it is also possible to identify which peak is the natural tune by comparison with low amplitude data points. At low amplitude, the beam samples fewer nonlinearities and therefore nonlinear resonances should be weaker. From there it is possible to identify the natural tune peak and avoid confusion with nonlinear resonances by reducing the range over which the peak finder will scan the spectrum around this initial guess. This is illustrated in Fig. 11 where it is clearly observed that for low oscillation amplitude a clean tune peak is observed around 0.325 . We can therefore conclude that the natural tune signal is around 0.325 and the line close to 0.324 corresponds to a nonlinear resonance. This resonance was indeed identified to be an octupolar resonance of the form $Q_{x}-Q_{y}-Q_{x D}+Q_{y D}=0.0$ (in Fig. 10, $Q_{x}=$ 0.3104, $Q_{x D}=0.32, Q_{y D}=0.334$ and $Q_{\text {res }}=0.3244$ ). The spectral line to the right of the bottom plot in Fig. 10 was identified to be another octupolar resonance.

Figure 12 shows the frequency of the spectral line associated to this resonance as a function of amplitude both from a direct analysis of the vertical turn-by-turn data and derived from the measured horizontal tune and ac dipole drive tunes for the data sets where it was possible to observe it. Good agreement is found between the two quantities confirming the hypothesis of the resonance.
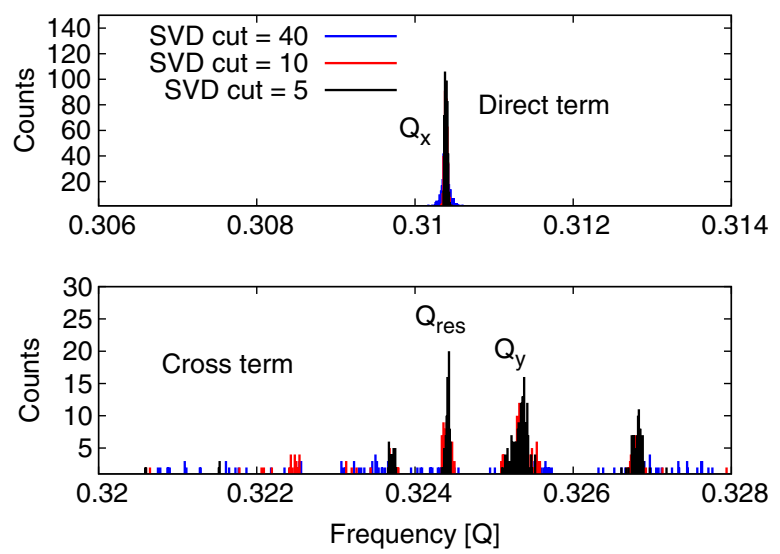

FIG. 10. Tune distribution of all LHC BPMs of the direct (top) and cross (bottom) terms of the amplitude detuning. While the direct term is cleanly measured and gives a single peak, several spectral lines appear on the cross term data making it difficult to identify the natural tune signal. 


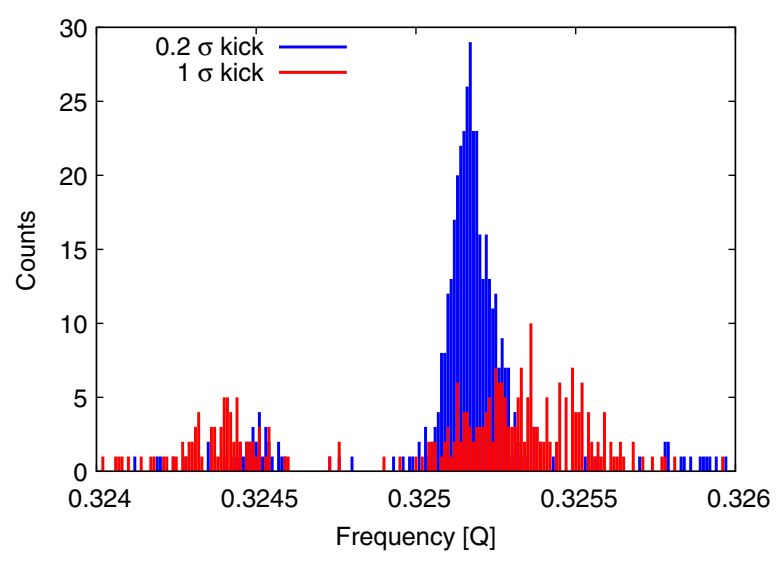

FIG. 11. Comparison of tune distribution for low amplitude and $1 \sigma$ oscillation amplitude which corresponds to the data set shown in Fig. 10. An SVD cut of 20 was performed for both data sets.

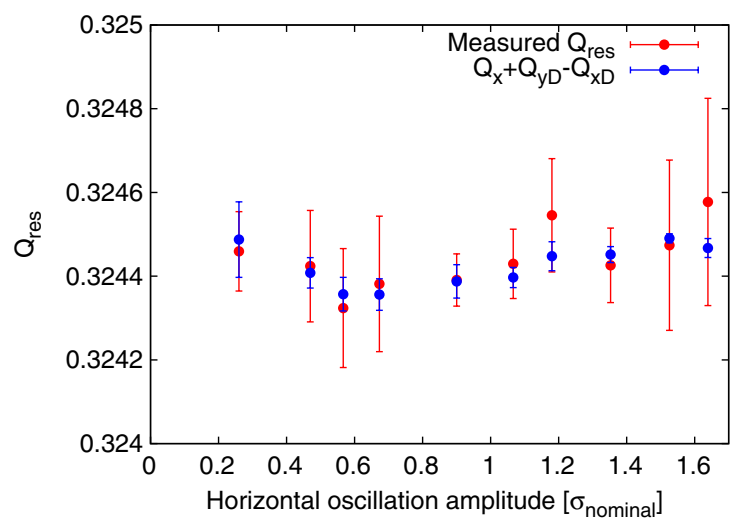

FIG. 12. Frequency of the spectral line $\left(Q_{\text {res }}\right)$ associated with the octupolar resonance $Q_{x}-Q_{y}-Q_{x D}+Q_{y D}=n$ directly measured from turn-by-turn data and derived from the measured horizontal natural tune and ac dipole drive tunes. $\sigma_{\text {nominal }}$ represents the LHC nominal rms beam size for an emittance of $3.75 \mu \mathrm{m}$.

Although the parasitic lines could be identified and the natural tune extracted these results also show that the beam was driven on or close to a resonance. This may significantly affect the results and make the theoretical approach presented above not appropriate. One should therefore be careful in the interpretation of the final results. Exciting the beam with an ac dipole in one plane only could partially mitigate the impact of nonlinear resonances by removing one of the four tunes from Eq. (36).

\section{Experimental results}

As mentioned in Sec. VIII A during these measurements the dominant sources of amplitude dependent detuning in the LHC (notably the Landau octupoles and the octupolar errors in the arc dipoles and the triplets) were either switched off or partially corrected. This leaves the smaller and less well understood errors to drive the detuning. This clearly compromises our ability to build an accurate model for comparison and makes the measurement more difficult by reducing the amplitude detuning. Nevertheless, our objective is to demonstrate the feasibility of this measurement at high energy by exciting the same beam multiple times. The comparison to the model is only done to verify the validity of the data. An accurate evaluation of the LHC multipole error and validation of the nonlinear model at top energy should be performed in a dedicated experiment.

During the top energy experiment, it was possible to acquire quality data sets only for Beam 2 horizontal excitations. The results are shown in Fig. 13. The amplitude of excitation in this case was limited to $2.5 \sigma$ due to losses in the final focusing triplets in IR1 and IR5 where the $\beta$ function reaches its maximum. Although this limit in aperture did not allow to reach very large amplitudes a clear detuning is observed for both planes. The cross term measurement proved to be very difficult due to the presence of nonlinear resonances and a low signal to noise ratio. However, a very clean measurement of the direct term of the amplitude detuning was achieved. Global coupling was well corrected during the experiment and no apparent cross talk between the horizontal and vertical driven actions was observed as seen in Fig. 7. To reproduce this behavior, it was decided to compare the data with the model without coupling and use the standard deviation of the distributions in Fig. 9 as error bars for the model. It is worth mentioning that the detuning coefficients without coupling are very close to the central values from the distributions in Fig. 9.

Referring to the theoretical results presented in Secs. III and IV of this paper, the octupolar contribution to the direct term of the amplitude detuning should be overestimated by
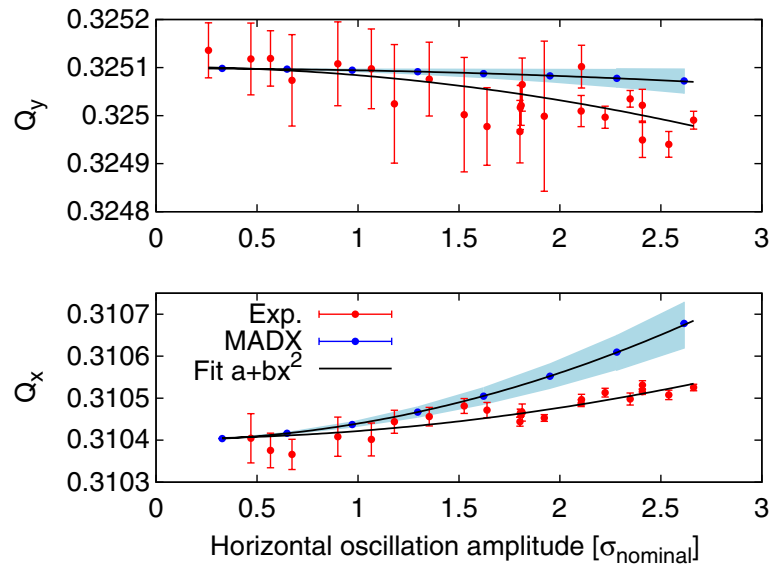

FIG. 13. Beam 2 amplitude detuning measured with horizontal ac dipole excitation for squeezed optics with $\beta^{*}=0.6 \mathrm{~m}$. The blue dots represent tracking from MAD-X using the experimental conditions. $\sigma_{\text {nominal }}$ represents the LHC nominal rms beam size for an emittance of $3.75 \mu \mathrm{m}$. The uncertainty due to coupling is illustrated by the light blue area. 
TABLE II. Octupolar term given by the fit results from data and MAD-X tracking with ac dipole and octupolar term from PTC which assumes free oscillations. The errors given in this table represent the fit errors. The uncertainties from coupling derived in Sec. VIII A are not included.

\begin{tabular}{lcc}
\hline \hline & $\partial Q_{x} / \partial 2 J_{x}\left[\mathrm{~m}^{-1}\right]$ & $\partial Q_{y} / \partial 2 J_{x}\left[\mathrm{~m}^{-1}\right]$ \\
\hline Data & $18528 \pm 10 \%$ & $-17387 \pm 23 \%$ \\
ac dipole model & $45757 \pm 1 \%$ & $-4492 \pm 2 \%$ \\
Free oscillation model & 22666 & -5493 \\
\hline \hline
\end{tabular}

a factor 2 ( $n=2$ for an octupolar field) when measured with an ac dipole while the cross term should not be affected. This is seen in Table II by comparing the free oscillation model (PTC) and ac dipole model (MAD-X tracking) and was verified experimentally at injection energy as presented in Sec. VII. Figure 13 shows the measured amplitude detuning and the one derived from MAD-X tracking with ac dipole using experimental working points and drive tunes. The fit results are presented in Table II.

Even in tracking simulations the cross term in the presence of the ac dipole deviates from theoretical prediction by approximately $20 \%$ whereas the direct term is consistent with predictions within error bars. This difference between tracking and theoretical expectation could be attributed to the excitation of nonlinear resonances which are also seen in simulations and indicates that the cross term measurement is strongly biased by these nonlinear resonances, in which condition the beam behavior is rather unpredictable. As shown in Sec. VIII A the cross term of the amplitude detuning also suffers significant uncertainty related to coupling. Under these conditions the cross term measurement interpretation is very difficult and should not be trusted. In future measurements, special care should be dedicated to coupling correction and working point optimization to reduce uncertainties, avoid resonances, and allow for a more meaningful measurement.

The direct term measurement appears to be rather clean and tracking simulations show a good agreement with theory. The uncertainty related to coupling is of the order of $20 \%$ which could be consistent with measurement errors. In this case, the disagreement between the measurement and the model can be assumed to be real and indicates an overestimation of approximately a factor 2.5 of the amplitude detuning in the model. The source of this discrepancy is attributed to the inaccuracy of the model with small detuning coefficient, i.e., after nonlinear corrections. The method remains however valid demonstrating the feasibility of amplitude detuning measurement with ac dipole at high energy.

\section{CONCLUSION}

The amplitude detuning equations in the presence of an ac dipole have been derived using a perturbation Hamiltonian for multipoles of order $2 n$. It has been demonstrated that a direct measurement of the amplitude detuning can be achieved using an ac dipole.

An experimental protocol has been defined and was applied to the LHC data to provide a first direct measurement of the amplitude detuning using an ac dipole. SVD cleaning of the raw data proved to be essential to measure the natural tune.

The theoretical derivations were demonstrated in the case of a detuning dominated by the octupolar term at LHC injection energy. The differences between the detuning coefficients measured with an ac dipole and a single kick are consistent with theoretical expectations.

The feasibility of the method at top energy by performing multiple large amplitude excitations on the same beam was demonstrated. Unfortunately, the experimental procedure was not optimized at the time and the measurement of the cross term may have been biased by nonlinear resonances. However, even under these conditions the measurements agree with model predictions within a factor 2.5 . Provided the difficulties to build a reliable nonlinear model for a machine as complex as the LHC, the discrepancy is most probably dominated by deficiencies from the model. This is nevertheless an excellent achievement for a first attempt and represents a demonstration of the feasibility of the method at top energy.

With some efforts, mostly in the direction of avoiding the excitation of nonlinear resonances (single plane ac dipole excitation, more adequate working point), and coupling correction, we believe that this method could become fully operational and provide an efficient and nondestructive tool to the measurement of amplitude detuning at high energy.

\section{ACKNOWLEDGMENTS}

The authors would like to thank the LHC operation and the participants to the machine development sessions for their support with the data acquisition. We would also like to thank M. Bai and M. Giovannozzi for carefully reading this paper and valuable discussions on nonlinear beam dynamics in the presence of ac dipole. This work was partially supported by Brookhaven Science Associates, LLC under Contract No. DE-AC02-98CH10886 and LARP with the U.S. Department of Energy.

[1] M. Bai, L. Ahrens, J. G. Alessi, K. Brown, G. M. Bunce, P. Cameron, C. M. Chu, J.W. Glenn, H. Huang, A.E. Kponou, K. Kruger, and W. Lamble, Phys. Rev. Lett. 80, 4673 (1998)

[2] R. Miyamoto, Ph.D. thesis, University of Texas, Austin, 2008.

[3] R. Tomás, O. Bruning, M. Giovannozzi, P. Hagen, M. Lamont, F. Schmidt, and G. Vanbavinckhove, Phys. Rev. ST Accel. Beams 13, 121004 (2010).

[4] S. Peggs and C. Tang, Report No. RHIC/AP/159, 1998. 
[5] R. Tomás, Phys. Rev. ST Accel. Beams 5, 054001 (2002).

[6] R. Tomás, M. Bai, R. Calaga, W. Fischer, A. Franchi, and G. Rumolo, Phys. Rev. ST Accel. Beams 8, 024001 (2005).

[7] R. Tomás, Phys. Rev. ST Accel. Beams 8, 024401 (2005).

[8] W. Herr and F. Schmidt, Report No. CERN-AB-2004-027ABP, 2004.

[9] R. Tomás, T. Bach, R. Calaga, A. Langner, Y. I. Levinsen, E. H. Maclean, T.H. B. Persson, P. K. Skowronski, M. Strzelczyk, and G. Vanbavinckhove, Phys. Rev. ST Accel. Beams 15, 091001 (2012).

[10] R. Calaga and R. Tomás, Phys. Rev. ST Accel. Beams 7, 042801 (2004).

[11] G. Vanbavinckhove, M. Aiba, R. Bartolini, R. Calaga, R. Miyamoto, M. Giovannozzi, F. Schmidt, R. Tomás, and E. Maclean, in Proceedings of the 2nd International
Particle Accelerator Conference, San Sebastián, Spain (EPS-AG, Spain, 2011).

[12] E. MacLean, S. Moeckel, T. Persson, S. Redaelli, F. Schmidt, R. Tomás, and J. Uythoven, Report No. CERN-ATS-Note-2013-022, 2013.

[13] T. Bach, M. Giovannozzi, A. Langner, Y. Levinsen, R. Miyamoto, E. Maclean, M. McAteer, S. Redaelli, P. Skowronski, R. Tomás, T. Persson, and S. White, Report No. CERN-ATS-Note-2013-015 (2013).

[14] P. Hagen, M. Giovannozzi, J.-P. Koutchouk, T. Risselada, F. Schmidt, E. Todesco, and E. Wildner, LHC Project Report No. 1123, 2008.

[15] F. Schmidt, in Proceedings of the 21st Particle Accelerator Conference, Knoxville, 2005 (IEEE, Piscataway, NJ, 2005).

[16] A. Boccardi, M. Gasior, R. Jones, P. Karlsson, and R. Steinhagen, CERN-LHC-Performance-Note-007, 2009. 